\title{
Deeply Virtual Compton Scattering at HERA II
}

\author{
Laurent Schoeffel
}

CEA Saclay, DAPNIA-SPP, 91191 Gif-sur-Yvette Cedex, France

\begin{abstract}
A new measurement is presented of elastic deeply virtual Compton scattering (DVCS) based on data taken by the H1 detector during the complete HERA II period. The data are well described by QCD based calculations. For the first time, a beam charge asymmetry is obtained in a colliding mode, using data recorded in $e^{-} p$ and $e^{+} p$. A significant non zero value is measured, related to the interference of QCD and QED processes, namely the DVCS and Bethe-Heitler reactions.
\end{abstract}

\section{Introduction}

The DVCS reaction, $\gamma^{*} p \rightarrow \gamma p$, can be interpreted as the elastic scattering of the virtual photon off the proton via a colourless exchange, producing a real photon in the final state. It has a clear experimental signature identical to that of the purely electromagnetic BetheHeitler $(\mathrm{BH})$ process. Since these two processes have an identical final state, it follows that they can interfere. The squared photon production amplitude is then given by [2]

$$
|\tau|^{2}=\left|\tau_{B H}\right|^{2}+\left|\tau_{D V C S}\right|^{2}+\underbrace{\tau_{D V C S} \tau_{B H}^{*}+\tau_{D V C S}^{*} \tau_{B H}}_{I},
$$

where $I$ denotes the interference term. For an unpolarised proton target and lepton beam, the interference term can be written quite generally as function of the azimuthal angle $\phi$, the angle between the plane containing the incoming and outgoing leptons and the plane defined by the virtual and real photon [2]

$$
I \propto-C\left[\cos \phi \operatorname{Re} \widetilde{\mathcal{M}}^{1,1}+\cos 2 \phi \operatorname{Re} \widetilde{\mathcal{M}}^{0,1}+\cos 3 \phi \operatorname{Re} \widetilde{\mathcal{M}}^{-1,1}\right]
$$

where $C= \pm 1$ is the lepton beam charge and $\mathcal{M}^{i, j}$ are related to helicity amplitudes [2]. Hence, cross section measurements which are integrated over $\phi$ are not sensitive to the interference term but the measurement of a cross section asymmetry with respect to the beam charge is a way to single out the interference term.

A general interest of the DVCS reaction lies in the the mass difference (skewing) between the incoming virtual photon and the outgoing real photon. This skewing can be interpreted in the context of generalised parton distributions (GPDs) [3] or in the dipole model framework [4]. In the following, new DVCS cross section measurements are presented and compared to QCD based models, extending previous analyses $[5,6]$. For the first time, a beam charge asymmetry is obtained in a colliding mode, using data recorded in $e^{-} p$ and $e^{+} p$, during the HERA II data taking.

\section{Data analysis and results}

The measurements of the DVCS cross section are based on the data collected by the H1 detector during the years 2004 till 2007, with HERA running with positrons/electrons colliding protons of energy 27.6 and $920 \mathrm{GeV}$. It corresponds to an integrated luminosity of $145 \mathrm{pb}^{-1}$ for each beam charge. To enhance the ratio of DVCS events w.r.t. BH ones, the 
photon is required to be detected in the forward or central region of the $\mathrm{H} 1$ detector, with a transverse momentum $P_{T}>2 \mathrm{GeV}$, while the scattered lepton is detected in the backward region, with an energy $E>15 \mathrm{GeV}$. To ensure the elastic selection and reduce the proton dissociation background, the absence of activity in the forward detectors is required [5]. To extract the DVCS cross section, the BH and inelastic DVCS backgrounds are subtracted bin by bin and the data are corrected for trigger efficiency, detector acceptance and initial state photon radiation.

Results are presented in figure 1. In figure 1 (left), we notice the reasonnable agreement of all analysis samples for the dependence of the DVCS cross section as a function of the centre-of-mass energy of the $\gamma^{*} p$ system, $W$. Using the electron sample only, the typical statistical and systematical errors on cross section are $10 \%$ and $15 \%$ respectively. Then, we can work with this electron sample for all analyses based on cross section.

In figure 1 (right), we observe the good description of $d \sigma_{D V C S} / d t$ by a fit of the form $e^{-b|t|}$. Hence, an extraction of the $t$-slope parameter $b$ is accessible for 3 values of the exchanged photon virtuality, $Q^{2}$ and $W$, extending the previous determinations [5]. The global value of $b$ is found to be $5.45 \pm 0.19 \pm 0.34 \mathrm{GeV}^{-2}$ at $W=82 \mathrm{GeV}$ and $Q^{2}=8 \mathrm{GeV}^{2}$. No dependence in $W$ is observed for $b$ and a significant $Q^{2}$ dependence can be extracted using also previous measurements at lower $Q^{2}[5]$. We obtain : $b\left(Q^{2}\right)=A\left(1-B \log \left(Q^{2} / 2\right)\right)$, with $A=6.98 \pm 0.54 \mathrm{GeV}^{-2}$ and $B=0.12 \pm 0.03$.
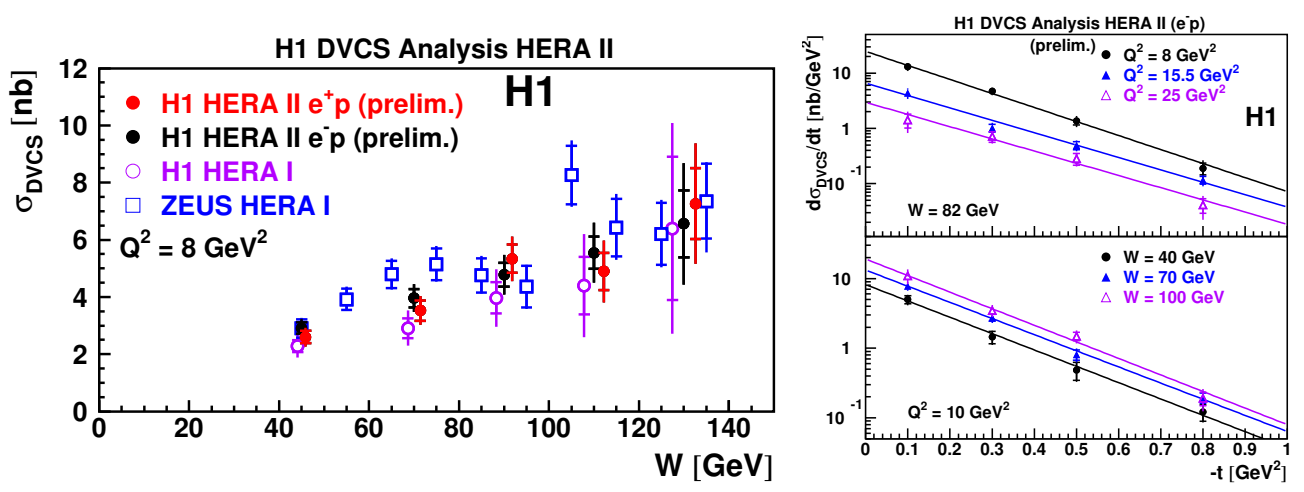

Figure 1: DVCS cross section for positrons/electrons samples as a function of $W$ (left) and differential in $t$, for 3 values of $Q^{2}$ and $W$ (right). The results of a fit of the form $e^{-b|t|}$ are also displayed.

\section{QCD Interpretations}

The DVCS cross section integrated over the momentum transfer can be written as

$$
\sigma_{D V C S}\left(Q^{2}, W\right) \equiv \frac{\left[\operatorname{Im} A\left(\gamma^{*} p \rightarrow \gamma p\right)_{t=0}\left(Q^{2}, W\right)\right]^{2}\left(1+\rho^{2}\right)}{16 \pi b\left(Q^{2}, W\right)}
$$

where $\rho^{2}$ is a small correction due to the real part of the amplitude and [3]. In the GPD formalism, the amplitude $\operatorname{Im} A\left(\gamma^{*} p \rightarrow \gamma p\right)_{t=0}$ is directly proportional to the GPDs. 
We define $S=\sqrt{\frac{\sigma_{D V C S} Q^{4} b\left(Q^{2}\right)}{\left(1+\rho^{2}\right)}}$, which is proportional to $\left|\operatorname{Im} A\left(\gamma^{*} p \rightarrow \gamma p\right)_{t=0}\left(Q^{2}, W\right)\right|$ and therefore directly contains information on the $Q^{2}$ evolution for the GPDs. The result is shown in figure 2 (left) an compared to a GPD model [3]. A reasonable description of the weak $Q^{2}$ dependence, compatible with a logarithmic behaviour, is observed for $S$.

The DVCS cross section can also be interpreted within the dipole approach [4]. It expresses the scattering of the virtual photon off the proton through its fluctuation into a color singlet $q \bar{q}$ pair (or dipole) of a transverse size $r \sim 1 / Q$. In the dipole approach the DVCS cross section is expected to verify the genuine property of geometric scaling [4]. This means that the cross section does not depend on both $x \simeq Q^{2} / W^{2}$ and $Q^{2}$ but obeys a scaling in a single variable $\tau=Q^{2} / Q_{s}^{2}$, where $Q_{s}(x)=Q_{0}\left(x / x_{0}\right)^{-\alpha / 2}$. Using parameters $Q_{0}=1 \mathrm{GeV}, \alpha=0.25$ and $x_{0}=2.710^{-5}[7]$, we can determine the variable $\tau$. The DVCS cross section is presented as a function of $\tau$ in figure 2 (right), which indicates that the geometric scaling property is verified within the present errors.
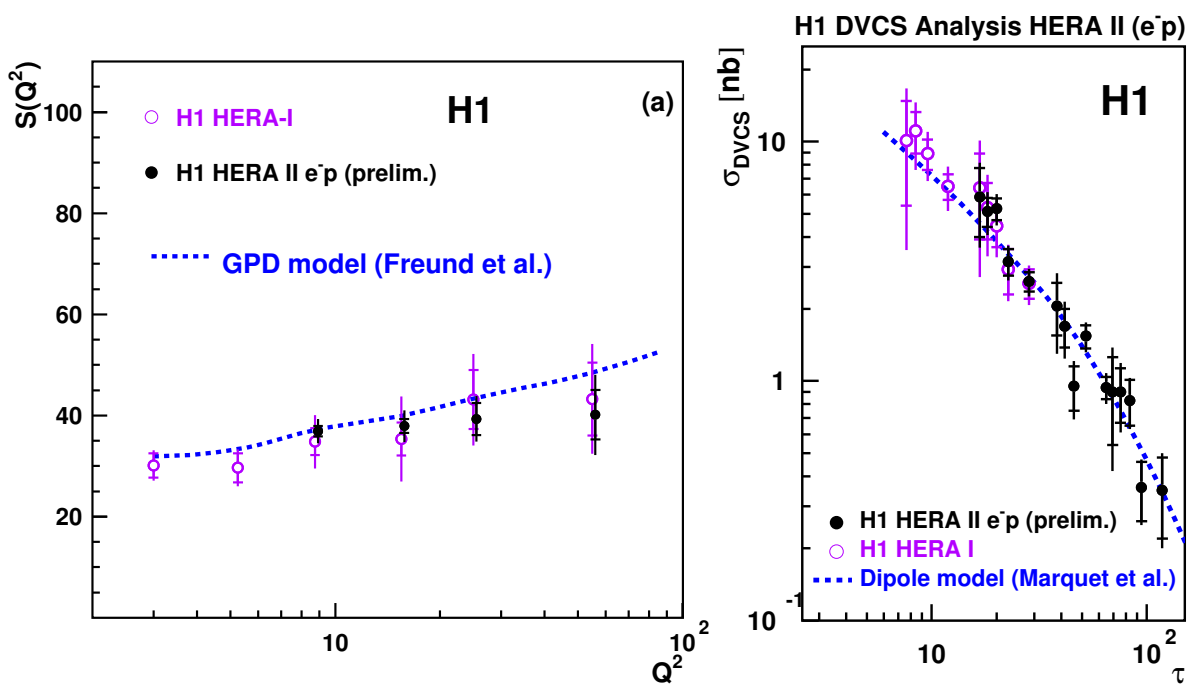

Figure 2: Observable $S=\sqrt{\frac{\sigma_{D V C S} Q^{4} b\left(Q^{2}\right)}{\left(1+\rho^{2}\right)}}$ with the prediction for the GPD model [3] (left). DVCS cross section measurements as a function of $\tau=Q^{2} / Q_{s}^{2}(x)$ with the prediction for the dipole model [4] (right).

\section{Beam charge asymmetry}

The determination of a cross section asymmetry with respect to the beam charge is realised by measuring the ratio $\left(d \sigma^{+}-d \sigma^{-}\right) /\left(d \sigma^{+}+d \sigma^{-}\right)$as a function of $\phi$. Note that $\phi$ is not defined when $|t|<|t|_{\text {min }}=x^{2} m_{p}^{2} /(1-x)$ [2]. However, the experimental resolution in $t$ is larger than the kinematical limit $|t|_{\min }$. Therefore we can not define $\phi$ when $|t|<0.05 \mathrm{GeV}^{2}$ and the BCA is measured only for $|t| \geq 0.05 \mathrm{GeV}^{2}$. In the expression of the BCA, $d \sigma^{+}$and $d \sigma^{-}$correspond to the DVCS cross section measured in positron and electron samples, over 
a bin $d \phi^{\text {a }}$. Results are presented in figure 3 with a fit in $\cos \phi$, which is expected to be the first dependence in $\phi$ following equation (2). After applying a deconvolution method to account for the resolution on $\phi$, the coefficient of the $\cos \phi$ dependence is found to be $p_{1}=0.17 \pm 0.03$ (stat. $) \pm 0.05$ (sys.).

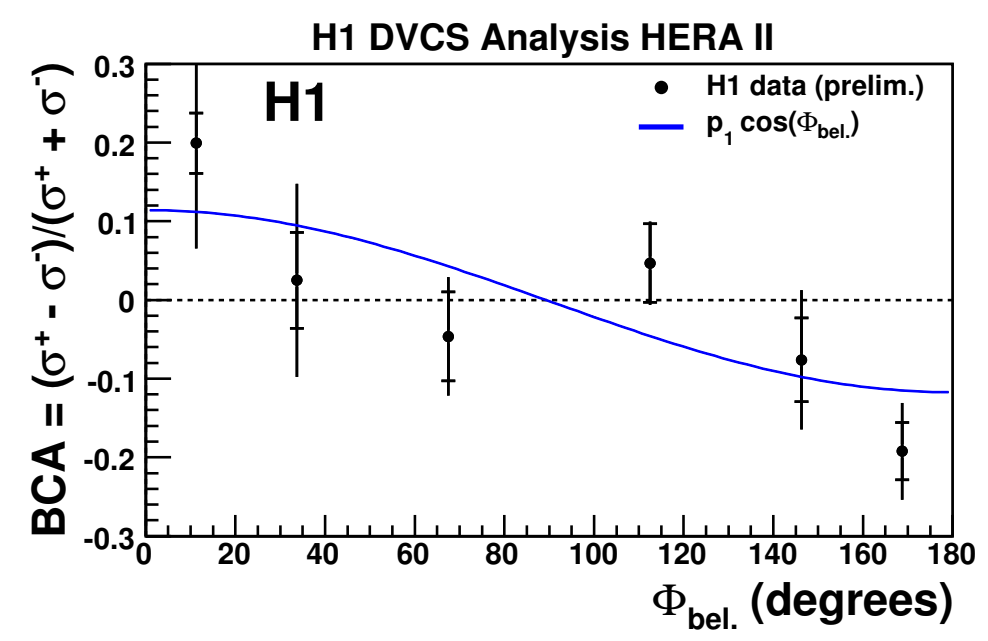

Figure 3: Beam charge asymmetry as a function of $\phi[2]$.

\section{Conclusion}

The DVCS cross section has been measured over a large kinematic domain using the complete HERA II data, extending previous analyses $[5,6]$. For the first time, a beam charge asymmetry is obtained in a colliding mode, using data recorded in $e^{-} p$ and $e^{+} p$. A significant non zero value is measured for $|t| \geq 0.05 \mathrm{GeV}^{2}$, which is related to the interference of DVCS and BH processes.

\section{References}

[1] Slides: http: //indico. cern. $\mathrm{ch} /$ contributionDisplay $\cdot$ py? contribId=92\&sessionId=7\& conf Id $=9499$

[2] M. Diehl, T. Gousset, B. Pire and J. P. Ralston, Phys. Lett. B 411 (1997) 193 [hep-ph/9706344] ; A. V. Belitsky, D. Mueller and A. Kirchner, Nucl. Phys. B 629 (2002) 323 [hep-ph/0112108].

[3] A. Freund, Phys. Rev. D 68 (2003) 096006 [hep-ph/0306012].

[4] C. Marquet and L. Schoeffel, Phys. Lett. B 639, 471 (2006) [hep-ph/0606079].

[5] A. Aktas et al. [H1 Collaboration], Eur. Phys. J. C 44, 1 (2005) [hep-ex/0505061].

[6] S. Chekanov et al. [ZEUS Collaboration], Phys. Lett. B 573, 46 (2003) [hep-ex/0305028].

[7] E. Iancu, K. Itakura and S. Munier, Phys. Lett. B 590 (2004) 199 [hep-ph/0310338].

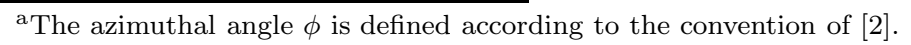

